\title{
Risques sanitaires globaux et politiques nationales : la gestion de la grippe aviaire au Vietnam
}

\author{
Muriel FIGUIÉ*, Tristan FOURNIER** \\ * Auteur correspondant : CIRAD-UMR MOISA, TA C 99-15, 73 rue JF Breton, \\ 34398 Montpellier Cedex 5, France \\ e-mail:muriel.figuie@cirad.fr \\ ** Université de Toulouse, CERTOP-CNRS, UTM, 5 Allées A. Machado, 31058 Toulouse \\ Cedex 9, France
}

Résumé - Nos sociétés sont actuellement confrontées à des risques sanitaires, souvent d'origine animale, qui amènent les organisations internationales telles que l'OIE, l'OMS et la FAO à promouvoir une nouvelle gouvernance mondiale. Nous examinons ici la question des spécificités nationales dans ce projet de gouvernance. Notre analyse est basée sur une étude menée en 2008 au Vietnam sur l'épizootie de grippe aviaire (H5N1). Nous distinguons, sur la période étudiée allant de 2003 à 2008, deux phases dans la gestion de ce risque par les autorités vietnamiennes : une première phase basée sur l'expérience nationale passée, notamment celle du SRAS, et répondant à des enjeux de légitimation interne du pouvoir central ; une deuxième phase plus conforme aux approches des organisations internationales et répondant à des enjeux de diplomatie internationale, liés en particulier au projet d'adhésion du pays à l'OMC. Cette analyse montre que le projet de gouvernance sanitaire mondiale ne doit pas sous-estimer les complexités au niveau local, que la considération des cultures nationales ne doit pas amener à négliger les dynamiques locales et que les contraintes ne doivent masquer les opportunités indissociablement liées aux risques.

Mots-clés : grippe aviaire, gouvernance, risque, santé, Vietnam

\section{Global health risks and national policies. Avian influenza risk management in Vietnam}

Summary - Our societies are facing risks for which international organizations such as the WHO, OIE, FAO call for a new international health governance. This paper aims at analyzing the confrontation between this international governance and national specificities.

Our analysis is based on empirical research conducted in Vietnam in 2008, on avian influenza (H5N1). It focuses on the period beginning with the first outbreaks in 2003 and ending in 2008. We bighlight two phases in H5N1 management by the Vietnamese authorities: a first phase based on past experiences (i.e. SARS) and related to national issues such as the consolidation of the central power in relation to provincial autborities; a second phase more in line with international governance principles and devoted to diplomatic issues such as WTO membership. This analysis concludes that international bealth governance should not underestimate the complexity of local situations, that taking into account national cultures of risk should not ignore local dynamics, and that focusing on constraints should not mask opportunities associated to risks.

Keywords: avian influenza, governance, bealth, risk, Vietnam

Classification JEL: I18, P29 


\section{Introduction}

Les crises sanitaires internationales récentes liées à l'émergence du virus du SRAS (Syndrome Respiratoire Aigu Sévère) ou aux virus grippaux (H5N1 ou H1N1) ont été accompagnées de la promotion par les organisations internationales, telles que l'OMS, l'OIE et la FAO ${ }^{1}$, d'une redéfinition des modèles de gestion des risques sanitaires. Se pose alors la question de la réception de ces modèles au niveau des différents Etats.

Cet article examine cette question dans le cas d'un pays du Sud, directement touché par une de ces crises, celle de la grippe aviaire (H5N1) et dépendant des ressources de la communauté internationale pour la gestion de cette crise: le Vietnam.

\subsection{Les nouveaux risques sanitaires et la communauté internationale}

Depuis une trentaine d'années, dans la communauté des chercheurs comme dans celle des experts, le vocabulaire utilisé pour qualifier les risques s'est considérablement enrichi : on y parle de risques modernes, majeurs, industriels, collectifs, systémiques, émergents, globaux... Cette prolifération du vocabulaire reflète le foisonnement des travaux sur ce thème. Elle exprime aussi un renouvellement et un élargissement de la conception du risque.

Dans la communauté des experts internationaux (OMS, OIE, FAO, OCDE $\left.{ }^{2}, \ldots\right)$, ces requalifications, malgré leurs nuances, reposent globalement sur un certain nombre de consensus. Ces experts constituent ainsi une communauté épistémique (Haas, 1992) partageant les mêmes points de vue sur la nature de ces risques et la nécessaire façon de les gérer:

- Ces risques émergent dans un environnement globalisé, de mobilité croissante et présentant des zones de très forte concentration humaine (OCDE, 2003; OMS, 2007). Un changement d'échelle est alors jugé nécessaire à l'appréhension de ces risques. Ce changement d'échelle s'exprime concrètement dans le contenu du Règlement Sanitaire International (RSI) de 2005 mis en place par l'OMS: le règlement de 2005 introduit le concept d' « urgence de santé publique à portée internationale » et encourage à dépasser les simples mesures locales de barrières aux frontières pour privilégier des mesures préventives à la source (OMS, 2007). En outre ce règlement encourage une meilleure coordination et concertation entre les actions des Etats, et pour une gamme de risques plus étendus (accidents industriels, résistance aux antibiotiques, bioterrorisme,...). Il traduit le passage vers une gestion davantage internationalisée et ouvre ainsi la voie à la légitimisation d'une ingérence sanitaire.

- Le caractère systémique reconnu de ces risques conduit à une volonté de désectorialisation de leur traitement. En particulier, les émergences et réémergences sanitaires et parmi elles l'épizootie de grippe aviaire (H5N1) ont contribué à l'adoption d'une stratégie commune par l'OIE, la FAO et l'OMS en

\footnotetext{
${ }^{1}$ OMS : Organisation Mondiale de la Santé ; OIE : Organisation Mondiale de la Santé Animale ; FAO : Food and Agriculture Organisation.

2 OCDE : Organisation de Coopération et de Développement Economiques.
} 
2008 : «Contributing to One world, One bealth $(\mathrm{OWOH})$ ». Cette stratégie marque selon le directeur général de l'OIE « la prise de conscience collective brutale du lien entre maladies animales et la santé publique » (Bernard Vallat, cité dans le bulletin de l'OIE, 2009). Plus largement, les problèmes d'émergences sanitaires sont désormais posés dans un contexte de changements climatiques, environnementaux, techniques, sociaux et économiques...

- Aux certitudes rassurantes fait place l'incertitude. Ainsi dans le document de l'OMS (2005) «Communication en cas de flambée épidémique » peut-on lire (p. 4) : «Les grandes inconnues: pourquoi ne peut-on apporter de réponses certaines aux questions qui se posent » et tout au long du texte: «peut être », « nous ne savons pas », « là encore nous l'ignorons », « personne ne peut le dire »... Cet aveu de mâtrise limitée se traduit par l'abandon d'objectifs d'éradication des risques sanitaires pour un objectif plus limité de préparation et de gestion.

- Un élargissement du système d'acteurs impliqués dans la gouvernance des risques s'opère: des solutions internationales, une gouvernance sanitaire mondiale s'imposent renforçant le rôle d'acteurs internationaux tels que l'OMS. L'objectif de désectorialisation amène à élargir les partenariats vers des partenaires non-traditionnels tels que les ONG environnementalistes, les zoos,... pour prendre l'exemple de l'OIE. L'élargissement se fait également au bénéfice du secteur privé, entreprises agro-industrielles, pharmaceutiques,... dans un mouvement de privatisation de la sécurité. Dans le secteur alimentaire, par exemple, les normes de qualité sanitaire privées se multiplient (comme par exemple la norme Eurepgap mise en place par la grande distribution).

En résumé donc, les modèles de gouvernance des risques sanitaires que cherchent à promouvoir la communauté des experts des organisations internationales citées reposent sur: une internationalisation, une désectorialisation, un objectif de gestion plutôt que d'éradication et une ouverture du système d'acteurs impliqués dans cette gouvernance.

\subsection{Les nouveaux modes de gouvernance des risques sanitaires à la lecture de la sociologie des risques}

Ces évolutions, que nous relevons ici au niveau international, reflètent ce que de nombreux chercheurs en sciences sociales ont identifié comme le passage de la gestion de « risques » à la gestion de "menaces »(Gilbert, 2002), de la gestion de "risques classiques » à la gestion de "risques nouveaux» (Godard et al., 2002), ou encore de la gestion de «risques de première modernité » à celle de "risques de deuxième modernité » (Beck, 2001), reconnaissant aux risques actuels une nouvelle nature ou insistant plutôt sur la nouveauté des modalités du processus social dont ils résultent.

Ces modalités sont jugées «plus ouvertes à la controverse, à la participation de multiples acteurs: autrement dit moins sous le contrôle des seuls experts et scientifiques» (Borraz 2008). Ainsi, l'étude de l'évolution des cadres d'analyses des risques avec le cas du Codex Alimentarius et du NRC Red Book (Millstone, 2009; Renn, 2008) confirme une ouverture accrue au public qui dépasse la seule phase de communication pour inclure progressivement la sélection des options de gestion, voire 
également la phase de définition des problèmes. S'il ne s'agit pas nécessairement, comme le souligne O. Borraz (2008), de modalités de définition du problème et d'intervention plus démocratiques que les précédentes, cette évolution témoigne néanmoins du fait que ces modalités ne relèvent plus nécessairement prioritairement de la puissance publique.

Ces travaux soulignent également l'incertitude qui caractérise ces risques, mais celle-ci ne saurait être réduite à une incertitude technique ou scientifique. Elle englobe également une incertitude relative aux conséquences sociales et à la capacité de contrôle du risque (Borraz, 2008). C'est ainsi leur potentiel de déstabilisation économique, social et politique qui caractérise ces nouveaux risques.

Concernant plus précisément l'internationalisation de la gouvernance des risques, divers auteurs (Douglas et Wildavsky, 1983; Renn, 2008) ont montré que les modes de gouvernance varient d'un pays à l'autre en fonction de facteurs contextuels tels que la capacité organisationnelle, la culture politique et règlementaire, la culture du risque et le climat social.

Le document stratégique «One World, One Health» postule l'existence d'un consensus et d'une vision commune autour de ces menaces, négligeant les différences politiques et les conflits d'intérêts : cette stratégie, selon Scoones et Forster (2008), est dominée par une vision du Nord focalisée sur la sécurité sanitaire (et privilégiant un cadrage du risque épidémique en termes de foyers, de crises et d'émergence) laissant de coté la question de l'inégalité structurelle d'accès aux ressources et d'exposition aux risques.

Dans un tel contexte, examiner les différences nationales dans les cultures et modes de gestion des risques et analyser comment elles s'influencent réciproquement devient un enjeu pour la recherche selon Taylor-Gooby et Zinn (2006). Or les travaux sont encore peu nombreux sur ce sujet et sont principalement centrés sur les pays développés : certains auteurs notent que les modes de gestion des risques tendent à converger entre les différents pays en conséquence de la globalisation (voir Löfsted et Vogel, 2001, à propos de l'Europe et des Etats-Unis) ou du fait du pouvoir des experts internationaux, membres d'un «collège invisible » capable de transformer des pratiques locales en normes internationales comme le montre Demortain (2009) à propos de la technique de l'HACCP (Hasard Analysis Critical Control Points) aujourd'hui internationalement utilisée. Dans le domaine plus général du transfert des politiques publiques, les travaux sont beaucoup plus nombreux et privilégient selon Hassenteufel (2005) trois questions (pourquoi? comment? avec quelle intensité ?) négligeant trop souvent trois aspects pourtant importants: la sociologie des acteurs opérant le transfert, les modes de réception des politiques publiques transférées (faisant référence au concept de traduction emprunté à Callon, 1986), et la façon dont se mêlent éléments transférés et éléments existants (l'hybridation).

\section{Objectif et méthode}

Notre objectif ici est d'alimenter ce débat en examinant les politiques de gestion de l'influenza aviaire $(\mathrm{H} 5 \mathrm{~N} 1)$ et du risque de pandémie grippale qui lui est associé, dans un pays du Sud, relativement pauvre: le Vietnam. C'est dans ce pays qu'a été 
enregistrée la réémergence du virus $\mathrm{H} 5 \mathrm{~N} 1$, en 2003 , après sa première apparition à Hong Kong en 1997. Nous essaierons surtout de comprendre pourquoi le Vietnam (pays encore très fermé il y a une vingtaine d'années), s'est montré coopératif au point d'être considéré comme le "bon élève » par la communauté internationale dans cette gestion du risque de pandémie (en opposition à la Chine ou à l'Indonésie par exemple). Nous faisons l'hypothèse que cette «exemplarité » du Vietnam est moins liée à sa maitrise du risque lui-même qu'aux réponses qu'il a su apporter aux pressions de la communauté internationale.

Nous décrirons d'abord deux phases distinctes dans la gestion de ce risque par les autorités vietnamiennes. Nous montrerons que ces deux phases correspondent au passage d'une gestion classique des risques à une gestion plus moderne, au sens où nous l'avons présenté plus haut. La succession de ces deux phases témoigne ainsi d'une convergence vers l'approche défendue par les organisations internationales. Nous montrerons que la traduction de la politique internationale au niveau de la politique vietnamienne s'est opérée à travers un dispositif institutionnel original, le PAHI (Partnership for Avian and Human Influenza). Nous montrerons également que loin de correspondre à une simple convergence, les modalités de gestion du risque $\mathrm{H} 5 \mathrm{~N} 1 \mathrm{au}$ Vietnam résultent d'un processus d'hybridation: se mêlent aux éléments externes, des éléments relatifs à l'histoire, au contexte local et surtout à des enjeux politiques spécifiques. L'évolution constatée correspond ainsi à deux cadrages distincts du risque : un premier cadrage en relation à des enjeux de légitimation interne du pouvoir central, un deuxième cadrage en relation à des enjeux de diplomatie internationale.

Cette recherche a été menée au printemps 2008 sur la base d'entretiens conduits au Vietnam auprès d'une vingtaine d'experts nationaux et internationaux travaillant sur la grippe aviaire. La moitié d'entre eux travaille dans des organisations vietnamiennes publiques et privées (représentants de divers départements du Ministère de l'Agriculture et du Développement Rural, du Ministère de la Santé, des services vétérinaires de plusieurs provinces, représentants du secteur privé). L'autre moitié regroupe principalement des acteurs de la communauté internationale intervenant au Vietnam (membres de l'équipe "grippe aviaire " de la FAO, experts des maladies parasitaires et infectieuses de l'OMS, représentants d'ONG étrangères, un représentant de l'hôpital français de Hanoi et des membres du PAHI - Partnership for Avian and Human Influenza).

De plus, ont été analysés différents documents stratégiques officiels du gouvernement («Red Book», «Green Book»...) ou des rapports d'experts (FAO, Agrifood Consulting International, AFD, AVSF), les comptes-rendus de réunions du PAHI, ainsi que la revue de presse réalisée par l'Ambassade de France à partir de différents quotidiens vietnamiens depuis 2003.

\section{Résultats}

Avant l'apparition du virus H1N1, en avril 2009 au Mexique, le risque majeur d'occurrence d'une pandémie grippale était associé au virus $\mathrm{H} 5 \mathrm{~N} 1$. Et, pour reprendre le langage militaire qui est souvent utilisé en pareil cas, le Vietnam était sur la ligne 
de front de la lutte contre la grippe. C'est en effet au Vietnam qu'ont été enregistrés les premiers décès humains liés à H5N1 en 2003 après ceux de Hong Kong en 1997. Et, à ce jour, le Vietnam comptabilise plus d'un $1 / 5$ des décès humains liés à $\mathrm{H} 5 \mathrm{~N} 1$ (on y comptabilise, depuis 2003, 112 cas humains de H5N1 dont 57 mortels, contre 471 cas dans le monde dont 282 mortels selon le site de l'OMS consulté le 2 février 2010).

La grippe aviaire y est aujourd'hui endozootique : sur les 59 provinces du pays, 27 ont déclaré des foyers de $\mathrm{H} 5 \mathrm{~N} 1$ chez les volailles en 2008 (dans 80 communes au total), et 18 provinces ( 75 communes) en $2009^{3}$. En ce sens, les résultats obtenus par le Vietnam en matière de lutte contre la grippe aviaire restent modestes.

Généralement, le récit de l'évolution de la grippe aviaire au Vietnam est centré sur les vagues épidémiologiques (ce qui confirme les propos de Scoones et Forster, 2008, cités plus haut) distinguant ainsi trois épisodes en fonction de la répartition spatiale et temporelle des foyers de grippe dans les élevages (Pfeiffer et al., 2007). Pour notre part, nous distinguons deux phases radicalement différentes dans leur origine et leur expression. La première phase démarre avec l'apparition officielle du virus sur le territoire vietnamien, la deuxième avec celle de l'apparition du virus en Europe.

\subsection{La gestion de la grippe aviaire par les autorités vietnamiennes : un effet «boomerang »}

\subsubsection{Phase I}

La première phase débute fin 2003-début 2004. Elle est liée à la situation épidémiologique locale marquée par le nombre de victimes humaines (jusqu’à 9 par mois) et par le nombre de foyers animaux (jusqu'à $25 \%$ des communes du pays déclarent des élevages touchés par le virus). La référence ici n'est pas la grippe espagnole comme en Europe, mais le SRAS (Syndrome Respiratoire Aigu Sévère) qui a sévi l'année précédente (faisant 5 morts au Vietnam, sur 63 cas, avant de tuer 774 personnes dans le monde, essentiellement en Chine). La presse titre alors «Une épidémie plus dangereuse que le SRAS » (article du journal Thanh Niên, quotidien de l'association de la jeunesse communiste, cité par Guénel et Klingberg, 2010).

Les autorités vietnamiennes et les organisations internationales avaient été critiquées au niveau international pour leur lenteur à répondre à l'épisode du SRAS. Dans le cas du virus H5N1, la réponse du gouvernement, appuyé par l'OMS, est plus rapide et massive : $17 \%$ des volailles sont abattues dans les trois premiers mois de 2004, soit 44 millions d'animaux (Delquigny et al., 2004) et une restriction des mouvements de volailles aux frontières des provinces et du pays est imposée.

"S'il y avait eu la grippe aviaire seulement, on aurait dit que l'OMS pétait les plombs, ouvrait grand le parapluie. Mais avant il y a eu le SRAS et l'OMS avait été très critiquée pour ne pas réagir assez vite » (médecin, coopération franco-vietnamienne).

\footnotetext{
3 D'après les bulletins du PAHI de janvier 2009 et de janvier 2010.
} 
Du côté des autorités vietnamiennes, c'est surtout le Parti Communiste Vietnamien (PCV), qui dans cette phase d'urgence prend les devants : il gère la crise par décrets, et s'appuie dans ses interventions (mesures d'abattages) sur l'armée et sur ce qu'on appelle le Front de la Patrie, lequel réunit les associations de masse (l'association des jeunes, l'association des femmes, l'association des paysans,...), (Guénel et Klingberg 2010 ; Vu Tuong, 2009). Le système d'acteurs mobilisé est le même que celui qui est traditionnellement mobilisé en cas de catastrophes naturelles (inondations, typhons...), ou même que celui mobilisé dans le passé en cas de conflits armés.

"Les vietnamiens sont tout à fait habitués et prêts à réagir à l'urgence: il n'y a pas de presse, pas d'opinion publique qui pourraient les embarrasser; pas d'intérêt sectoriel à ménager et pas de complexe. Et la chaine de commande - l'armée et le Parti-est efficace » 4 (expert, Organisation internationale).

Pendant ce temps, les pays occidentaux adoptent des stratégies nationales de protection, de portée limitée (stockage d'antiviraux et des masques; Gilbert, 2007). Le Vietnam considère alors que la disparition de la grippe est l'affaire de quelques mois, comme cela avait été le cas du SRAS. Le Gouvernement déclare en mars 2004, contre l'avis de l'OMS qui invite à la prudence, que le virus a été éradiqué de son territoire. Mais, donnant raison à l'OMS, de nouveaux foyers se déclarent dans les semaines qui suivent. Pourtant l'activité politique et médiatique est retombée et ne reprendra de l'ampleur que fin 2005.

\subsubsection{Phase II}

A partir du dernier trimestre 2005, une deuxième impulsion est donnée au traitement du problème. Cette impulsion n'est plus liée comme précédemment à la dynamique du virus sur le territoire vietnamien ou à d'autres évènements nationaux. Elle a une origine externe.

Depuis le début de la crise, fin 2003, la présence du virus a été confirmée dans une dizaine de pays, principalement en Asie. Mais au cours du dernier semestre de 2005 seulement, dix nouveaux pays sont touchés (la présence du virus y est identifié chez des animaux domestiques ou sauvages) dont la Russie, la Turquie, la Roumanie, la Croatie, et le Royaume-Uni. Des premiers cas humains apparaissent en Turquie en janvier 2006. En février 2006, des élevages sont touchés en France et le virus est identifié chez des oiseaux sauvages en Bulgarie, en Italie, en Slovénie, en Grèce et en Allemagne. Un chat domestique meurt en Allemagne victime du virus. Le même mois, l'Afrique est à son tour touchée (OMS, 2010). La tension s'accroît dans le monde occidental à mesure que le virus progresse.

Le Congrès américain engage en 2005 une évaluation de l'action de la communauté internationale et des principaux pays touchés par le virus dont le Vietnam. Cette évaluation souligne de très nombreuses déficiences dans la surveillance

\footnotetext{
${ }^{4}$ Lors de sa visite à Hanoi, en 2005, Xavier Bertrand, alors Ministre de la Santé du gouvernement français, explique avec regret à son homologue vietnamien, et devant l'un de nos enquêtés, qu'il ne dispose malheureusement pas en cas de crise sanitaire d'une telle marge de manœuvre, notamment vis-à-vis du Ministre de l'Agriculture.
} 
et le contrôle de la maladie. Se développe alors le constat, parallèlement aux leçons tirées de la menace terroriste, que les USA ne peuvent se contenter pour leur protection d'une action sur leur territoire mais doivent s'engager au niveau international (Congressional Research Service, 2006).

Au Sommet Mondial de l'ONU, en septembre 2005, le Président des Etats-Unis, G. Bush, annonce alors la décision de son pays de s'investir dans la coordination mondiale de la lutte contre la grippe aviaire en même temps qu'il exige la transparence des pays sur leur situation épidémiologique. En s'accordant sur le nouveau règlement sanitaire international de 2005, la communauté internationale (et les pays du Nord surtout) entérine un changement de stratégie: il faut traiter le mal à sa source, légitimant ainsi une intervention plus directe de la communauté internationale, au Vietnam notamment. Un coordinateur international pour la grippe aviaire et humaine est nommé par le secrétaire général de l'ONU en septembre 2005, marquant la volonté d'internationaliser la gestion de ce risque. Cette prise de conscience et ce changement de stratégie dans les pays occidentaux ont un effet boomerang sur le Vietnam désormais soumis à une pression internationale accrue.

Si l'OMS avait déjà renforcé son intervention au Vietnam à l'occasion du SRAS, c'est au tour de la FAO d'intensifier sa présence. Les Nations-Unies organisent la coordination des bailleurs de fonds et exigent, mi-2005, une implication plus forte de la haute hiérarchie vietnamienne avec notamment un dialogue direct avec le Premier Ministre vietnamien.

Les coopérations bilatérales se multiplient avec une présence accrue sur le terrain vietnamien : c'est le cas des USA (via le Center for Disease Control notamment) mais aussi des coopérations japonaises (surtout investies dans la communication) et néozélandaises. Et c'est à travers ces organisations internationales et étrangères, que les ONG multiplient leurs interventions (Association Vétérinaires Sans Frontières, Academy for Educational Development, CARE...).

"Les ONG ont beaucoup bénéficié financièrement de la grippe aviaire... Et votre institut de recherche aussi » (expert, PAHI).

Le Vietnam est mis sous pression pour préparer un plan d'action plus large qui englobe une stratégie en cas de pandémie, plan qu'il devra présenter à la première conférence internationale pour le financement de la lutte contre le virus de la grippe aviaire, à Pékin en janvier 2006, pour obtenir une partie des fonds que la communauté internationale promet de mobiliser (1,9 milliards de $\$$ au total).

Les déclarations officielles du gouvernement vietnamien et le nombre d'articles de presse consacrés à la grippe aviaire connaissent un nouveau pic. Et ceci sans que la situation épidémiologique locale ne connaisse de véritable changement. Le Premier Ministre vietnamien répond à la pression des Nations-Unies en s'adressant pour la première fois directement à l'ensemble du pays à travers un télégramme officiel ${ }^{5}$ largement diffusé par les médias dans lequel il annonce le «renforcement des mesures de prévention et de lutte contre la grippe aviaire ». Les marchés de volailles vivantes

5 Ce télégramme est identifié par le code $\mathrm{N}^{\circ}$ 1686/TTg-NN. 
sont interdits en ville où seuls pendant quelques mois les supermarchés sont autorisés à commercialiser les volailles. Il est demandé aux personnes résidant en ville de tuer leurs oiseaux de compagnie: ces oiseaux sont très nombreux au Vietnam; les propriétaires préferent alors les libérer dans les parcs de la ville. Le Vietnam annonce par ailleurs sa volonté de favoriser une restructuration du secteur avicole ${ }^{6}$ en faveur d'élevages plus modernes jugés plus sûrs (en référence au concept de plus en plus mobilisé de biosécurité) et qu'il tente sans grand succès de mettre en œuvre à travers une politique de relocalisation des élevages (MARD, 2007). Et surtout, une vaste et ambitieuse campagne de vaccination est lancée à l'échelle nationale.

\subsection{Le passage d'une gestion classique à une gestion moderne}

La description faite plus haut de l'évolution de la politique de gestion de la grippe aviaire par les autorités vietnamiennes, entre les phases I et II, peut être analysée autour des points suivants :

\subsubsection{De l'éradication à la gestion, de l'urgence à la préparation}

Dans la première phase, l'objectif est d'éradiquer le virus. Dans ce but, les volailles sont abattues en masse (certains experts évoqueront une "panique » du gouvernement). Cette mesure est la plus impopulaire de celles prises par le gouvernement. La stratégie de communication du gouvernement, jugée trop alarmiste par les éleveurs, lui est également reprochée. Elle aurait amplifié les conséquences économiques de la grippe en détournant les consommateurs de la consommation de volailles. Les autorités reviennent sur ces mesures qui se sont révélées mal adaptées et couteuses pour le gouvernement et les éleveurs: fin 2005, les autorités adoptent un objectif non plus d'éradication mais de gestion d'un virus endémique, l'éradication étant reportée à un futur plus lointain. Le gouvernement fait état de ce changement de stratégie dans le bilan qu'il dresse en 2010 de sa gestion de la grippe aviaire: "A l'époque [en 2004], l'abattage massif était considéré comme la meilleure approche pour contrôler la maladie et éliminer le virus... L'objectif à court et moyen termes au Vietnam devient en 2005 la réduction du niveau de circulation du virus. L'objectif de long terme reste l'élimination du virus H5N1 HPAI des élevages de volailles vietnamiens. Mais nous sommes réalistes, nous savons que ceci prendra du temps » (MARD et $\mathrm{MOH}, 2010)$. Le Vietnam va plus loin encore dans la réduction de ses ambitions, en se préparant sous la pression internationale, à l'échec en quelque sorte avec l'élaboration du plan de préparation à la pandémie grippale ${ }^{7}$.

\subsubsection{Elargissement du système d'acteurs : Parti Communiste Vietnamien, associations de masse et secteur privé}

"Tout d'abord c'est le Secrétariat du Parti qui a promulgué des directives et a mobilisé l'ensemble des forces du Pays. Ensuite il y a eu les règlementations

\footnotetext{
6 Il s'agit de la décision QD 394 du Ministère de l'Agriculture.

7 Il s'agit du Red Book (Integrated national plan for avian influenza control and human pandemic influenza preparedness and response, 2006-2008) et du Green Book (Integrated national operation program for avian and human influenza, 2006-2010), publiés respectivement en janvier et mai 2006.
} 


\section{du Gouvernement, les subventions,... » (membre du Ministère de l'Agriculture et du Développement Rural).}

En effet, le passage d'une gestion d'urgence à la préparation du plan de pandémie grippale impose une plus grande mobilisation du Ministère de la Santé, aux côté du Ministère de l'Agriculture et du Développement Rural déjà très fortement impliqué. Le système d'acteurs s'élargit également au secteur privé : ce secteur, peu présent dans la première phase d'éradication du virus, est ensuite largement sollicité à travers les entreprises du secteur agro-alimentaire. Cette phase est l'occasion au niveau national de pousser un projet antérieur à l'émergence de la grippe aviaire, celui de la modernisation du secteur de l'élevage : si le marché est approvisionné à $80 \%$ par des petits élevages de volailles de type familial, le projet du Gouvernement est que, dans un délai de dix ans, il soit approvisionné à $80 \%$ par un secteur moderne industriel, jugé plus sûr d'un point de vue sanitaire (MARD, 2006 cité par Agrifood Consulting International, 2007). A travers les règlementations, les nouvelles normes sanitaires, les politiques de crédit et même les politiques de réallocations foncières, le gouvernement appuie la filière avicole industrielle. Et l'Etat délègue même au secteur privé une fonction considérée comme régalienne (surtout dans un pays communiste: la protection de la santé de la population). "Si vous ne voulez pas être malade, achetez vos volailles au supermarché », annonce publiquement le Ministère de la Santé à la radio.

Les petits éleveurs sont encouragés au début de la crise à changer d'activité et l'élevage de canards est même interdit. Par la suite, le gouvernement a assoupli sa position considérant que la stratégie ne doit pas se faire au détriment des petits élevages et a entrepris de les associer à la lutte contre la grippe (MARD et $\mathrm{MOH}$, 2010). Pour faire face aux difficultés de l'Etat à encadrer cette vaste communauté d'éleveurs, le Ministère de l'Agriculture compte sur la contractualisation avec le secteur privé (notamment avec le groupe agro-industriel thaillandais Charoen Pokphand).

\subsubsection{La vaccination: décider en situation d'incertitude}

Le Vietnam est le premier pays à avoir opté pour une vaccination de masse des volailles contre le virus $\mathrm{H} 5 \mathrm{~N} 1$. La décision de la vaccination, selon nos entretiens auprès de la FAO et du Ministère de l'Agriculture, a été l'une des décisions les plus difficiles à prendre au cours de cette crise. Les avis des experts étrangers au chevet du Vietnam divergeaient quant à l'opportunité d'une telle mesure. La vaccination de masse des volailles permet de réduire la circulation du virus dans les populations de volailles et de réduire ainsi sur le court terme les risques de transmission du virus aux hommes. Mais la vaccination de masse, pour être efficace sur le long terme et dans le contexte d'élevages de basse cour, exige des moyens humains, financiers et organisationnels rarement disponibles dans les pays en développement. Elle a également un certain nombre d'inconvénients (réduction des signes cliniques retardant la détection des foyers, augmentation des risques de dérive génétique,...) qui alimentent la controverse au sein du monde des experts sur son opportunité aussi bien du point de vue de la santé animale que de la santé humaine (Hinrichs et al., 2010).

En réaction aux débats et controverses auxquels ils assistent, les décideurs vietnamiens confient leur déception quant au soutien reçu des experts étrangers dans 
cette prise de décision. Les autorités vietnamiennes tranchent alors en faveur de la vaccination, non pas sur des bases scientifiques, alors encore fragmentaires, mais dans un objectif de communication : il s'agit de faire savoir à la nation et au reste du monde que le gouvernement est actif dans la lutte contre le virus. Le Vietnam devient alors un pays « test » pour la communauté internationale.

\section{"Le Vietnam au début a été le cobaye; tout le monde observait ce qu'il s'y passait» (expert, Organisation internationale).}

\subsection{L'interface avec le niveau international}

\subsubsection{Stabilité et transparence}

Le gouvernement vietnamien a une tradition de communication qui vise avant tout à rassurer sa population, assumant ainsi le rôle d'un état protecteur et paternaliste: il est courant d'entendre dire dans la population qu'il peut être nécessaire au gouvernement de mentir si cela permet de protéger le peuple et d'éviter la panique. C'est sur ses résultats que le gouvernement doit être jugé. Ainsi, selon un chercheur spécialiste de la grippe en Asie:

"Nous sommes ici dans un pays communiste. L'objectif principal est d'assurer la stabilité. Ici l'Etat est source de confiance. Ce n'est pas comme en France ou au Royaume-Uni par exemple où il y a toujours une méfiance à l'égard du gouvernement et où la transparence est importante... L'objectif principal ici est d'assurer la stabilité. Alors le gouvernement peut ne pas tout dire ; il peut exagérer son niveau de maitrise du problème... L'Europe est venue ici avec une perspective très différente: nous sommes venus ici pour analyser de façon scientifique le problème de la grippe, pour définir une stratégie... Mais la perspective du gouvernement est tout autre : il vise à assurer la stabilité du pays et sa réputation».

Si ce commentaire tend à sous-estimer les enjeux politiques associés à l'intervention internationale, il souligne parfaitement la diversité des enjeux et des systèmes de valeurs que le projet de gouvernance internationale des risques sanitaires amène à confronter.

A l'époque du SRAS, les organisations internationales avaient reproché aux autorités vietnamiennes une certaine opacité. En particulier, des cas auraient été dissimulés pour ne pas compromettre la tenue au Vietnam fin 2003 des XXIIe Jeux d'Asie du Sud-Est (Southeast Asian Games ou SEA Games), rencontres sportives de l'Association des Nations de l'Asie du Sud-Est, ASEAN. Aujourd'hui les experts sont unanimes pour souligner la coopération dont fait preuve le Vietnam dans le domaine sanitaire, la rapidité en particulier avec laquelle les informations (concernant de nouveaux cas ou foyers) sont communiquées. Ainsi un des experts de l'OMS interrogés dit avoir été très impressionné par la rapidité nouvelle avec laquelle en 2008 le Vietnam a déclaré, après seulement quelques cas, des foyers de choléra à la communauté internationale. Il semble donc que le Vietnam ait pris en compte au cours de la gestion de la crise la valeur de la transparence sur la scène internationale, même si la transparence est beaucoup moins valorisée sur la scène nationale. 


\subsubsection{Les enjeux politiques nationaux et internationaux pour le Vietnam}

Les enjeux politiques associés à la maitrise du virus H5N1 sont, pour le Vietnam, à la fois des enjeux de stabilité sur la scène nationale et de réputation sur la scène internationale.

La première phase de gestion de la grippe aviaire apparait essentiellement au service des enjeux nationaux: le virus se trouve mis au service d'une centralisation du pouvoir et d'un projet nationaliste. En effet, depuis les réformes de 1986 (qui visent à une libéralisation de l'économie), les autorités provinciales ont accru leur pouvoir, en particulier économique (par rapport au pouvoir central). A l'occasion de la grippe aviaire, comme le montre Vu Tuong (2009), la gestion de H5N1 est l'occasion de réaffirmer le pouvoir central indépendamment des réalités locales (comme par exemple à travers les mesures autoritaires d'abattage massif). C'est également l'occasion pour le gouvernement de mettre tous les dysfonctionnements de la gestion de cette crise sur le compte de l'incompétence et de la corruption des autorités locales. En outre, l'analyse de la presse (Guénel et Klingberg, 2010 ; Vu Tuong, 2009) montre que la victoire contre la grippe y est présentée comme une question d'honneur national, et la lutte entreprise contre le virus donne au gouvernement la possibilité de mobiliser les masses derrière le Parti contre un nouvel ennemi commun.

En revanche dans la deuxième phase de la gestion de la grippe aviaire, le virus semble être mis au service d'autres objectifs, lesquels se jouent sur la scène internationale. Car, comme l'affirme un des responsables du Ministère de la Santé interrogé en 2008 :

"C'est une question qui concerne notre pays mais aussi l'ensemble des pays du monde. Il nous faut partager cette responsabilité. Certes la destruction des poulets cause des pertes économiques importantes à notre population, en particulier aux éleveurs des régions pauvres... Sans ces poulets, certaines familles ne peuvent peut-être plus scolariser leurs enfants. Mais le Gouvernement est déterminé et fait preuve ainsi d'une grande responsabilité à l'égard du monde entier».

La réactivité du Vietnam à la deuxième impulsion envoyée par la communauté internationale est à interpréter comme un élément d'une stratégie d'intégration à la communauté internationale initiée il y a plus de vingt ans. Car le Vietnam revient de loin : en effet, en conséquence de l'invasion du Cambodge en 1978, le Vietnam déjà sous embargo commercial et financier américain depuis 1974 est boycotté par la communauté internationale en 1987. Par ailleurs, à la même période, il perd un appui sérieux avec l'effondrement de l'URSS. La situation économique intérieure est catastrophique (et se traduit par d'importantes famines en 1989). Pour surmonter les crises, le Vietnam a besoin d'élargir son partenariat international et de renoncer ainsi à une vision dichotomique du monde constitué de pays amis et pays ennemis. Cependant à l'époque, «regagner la confiance (internationale)», note Do Hien (2007: 271), «constituait pour le Vietnam une mission quasi impossible». Le Vietnam pourtant y parvient et dans un temps relativement bref. Le pays entreprend d'abord de normaliser ses relations avec la Chine (avec la fin du conflit au Cambodge) et d'intensifier ses relations avec ses voisins d'Asie du Sud-Est. Le Vietnam fait un geste important en acceptant de collaborer avec les USA sur le projet MIA (Missing In Action). Ce geste 
conduit à une levée de l'embargo commercial américain, lui permet d'accéder à de nombreux prêts internationaux (Banque Asiatique de Développement, Fond Monétaire International,...) et contribue probablement à accélérer l'admission du Vietnam comme membre de l'ASEAN, l'Association des Nations de l'Asie du Sud-Est (Do Hien, 2007). Cette admission n'est pour le Vietnam qu'une première étape de sa stratégie d'intégration de la communauté internationale avec en ligne de mire son adhésion à l'Organisation Mondiale du Commerce (OMC).

Or, nous constatons qu’à la période de la crise de la grippe aviaire, les Etats-Unis apparaissent comme le principal obstacle à l'entrée du Vietnam à l'OMC en même temps qu'ils s'impliquent fortement à partir de 2005 dans la lutte internationale contre le virus. Les visites de représentants officiels du gouvernement américain qui se succèdent au Vietnam dès 2005, jusqu'à celle du Président G. Bush lui-même en novembre 2006, montrent comment aux yeux des autorités américaines les deux points sont liés ${ }^{8}$. En réponse à ces pressions, le Vietnam a à cœur de se montrer un «bon citoyen du monde » (Good Global Citizen) : un virus ne doit pas compromettre plus de 20 ans d'efforts pour la réintégration du Vietnam dans les circuits diplomatiques internationaux.

"Le Vietnam a longtemps été considéré comme un ennemi du monde. Depuis 15 ans il mène une stratégie de réintégration à la communauté internationale. Quand le Vietnam a réalisé la dimension globale du risque il a très vite réagi. C'est vrai qu'ils se sont un peu trompés en 2004, mais ça n'a pas duré » (expert, Organisation internationale).

\subsubsection{Un espace de coordination et de " mise à distance » de la coopération internationale: le PAHI}

Pour gérer la grippe aviaire, les autorités vietnamiennes réactivent dès 2004 le Comité National de Coordination (NSC) mis en place l'année précédente à l'occasion de l'épisode du SRAS. Ce NSC permet de coordonner les activités des différents ministères impliqués dans la gestion du virus (Ministère de l'Agriculture et du Développement Rural et Ministère de la Santé surtout, mais aussi Ministère du Commerce, Ministère des Finances, Armée Populaire Vietnamienne,...) et participe du projet de désectorialisation du traitement de ces risques ${ }^{9}$.

Mais un autre défi est aussi de coordonner les activités foisonnantes de la communauté internationale. C'est le rôle du PAHI (Partnership for Avian and Human Influenza), créé en 2005. Son rôle n'est cependant pas limité à cette coordination. Les organisations étrangères étant exclues du NSC afin de marquer la souveraineté du Vietnam, le PAHI sert en quelque sorte de point de contact entre les autorités vietnamiennes et les organisations étrangères ou internationales.

"Le fonctionnement du NSC montre la souveraineté du Vietnam... Ce n'est pas comme au Laos où les organisations internationales sont très présentes et très puissantes par rapport au gouvernement. Le Vietnam est un pays souverain et la FAO n'a participé qu'une seule fois à une réunion du NSC

\footnotetext{
8 Voir par exemple la page du VietNam Net Bridge datée du 1/02/2006.

9 Depuis le NSC traite également d'autres maladies infectieuses comme celle liée au virus H1N1.
} 
parce que le Gouvernement l'y avait invitée pour un exposé. Le gouvernement vietnamien veut insister sur le fait qu'il est très ouvert, prêt à donner des informations, mais cela ne veut pas dire que les organisations internationales peuvent intervenir au NSC. C'est au travers du PAHI que les informations sont échangées avec les organisations internationales » (expert, PAHI).

Participent au PAHI les représentants des différents ministères et des différentes ambassades. Le statut des participants (ministres et ambassadeurs ou simples subalternes) ainsi que la fréquence des réunions donnent le pouls de la situation de crise. En même temps que le PAHI apparait comme un instrument de mesure de la crise dans sa dimension internationale, il apparait aussi comme un lieu de construction d'une communauté épistémique entre experts vietnamiens et étrangers (ainsi qu’à l'occasion des très nombreuses conférences qui se tiennent régulièrement d'hôtels internationaux en hôtels internationaux), mais aussi s'y joue une mise en politique du risque à l'interface du national et de l'international.

L'existence du PAHI témoigne à la fois de la transparence jouée par le Vietnam à l'égard de la communauté étrangère en même temps qu'il met à distance cette communauté pour mieux asseoir sa légitimité. Les représentants des autorités vietnamiennes rencontrées à l'occasion de cette recherche se plaisent à souligner que le Vietnam n'est ni le Laos car il n'a pas renoncé à l'occasion de la grippe à sa souveraineté, comme déjà cité, ni la Chine car le Vietnam s'est montré transparent, ni l'Indonésie car il a joué le jeu de la coopération.

\section{Conclusions}

Au terme de cette étude, deux périodes dans l'historique de la crise de grippe aviaire au Vietnam ont pu être identifiées. Une première phase, qui s'étire du début de la crise fin 2003 jusqu'à l'automne 2005 et est notamment caractérisée par le rôle prédominant de l'Etat, appuyé par les associations de masses traditionnelles (l'Union des Femmes et l'Union des Fermiers notamment) et le Parti Communiste. Le but premier est d'éradiquer le virus $\mathrm{H} 5 \mathrm{~N} 1$ et, ce faisant, de renforcer le pouvoir et la légitimité du gouvernement vietnamien aux yeux de la population locale et de la Communauté Internationale. Une seconde période démarre suite à l'apparition du virus en Europe fin 2005 et est marquée par l'implication de nouveaux acteurs : la coopération bilatérale, le secteur agro-industriel privé, les $\mathrm{ONG}, .$. Le risque est dès lors considéré comme endémique et sa gestion relève non plus de l'urgence mais de changements structurels sur le long terme dans l'organisation des services de santé et de la filière avicole. Le risque s'inscrit dès lors dans le fonctionnement de la société. Sa gestion est jugée non plus sur les résultats obtenus mais sur les moyens mis en œuvre pour le contenir. Par la même occasion, les autorités mettent le virus au service de sa diplomatie internationale.

L'exemple du Vietnam, de la façon dont les autorités ont géré la crise de la grippe aviaire, permet d'illustrer le poids des spécificités nationales dans la gestion des risques et dans les enjeux qui lui sont liés.

"La rapidité de réponse du Vietnam s'explique par son bistoire. Le pays a dû mobiliser ses citoyens de nombreuses fois dans l'bistoire. Je suis née en temps 
de paix mais je suis imprégnée de cette culture vietnamienne de lutte collective » (membre du Ministère de l'Agriculture et du Développement Rural).

Le Vietnam au delà du risque sanitaire a dû gérer des risques politiques. O. Borraz (2008) souligne le potentiel de déstabilisation politique des risques modernes lié au fait que ces risques interrogent la capacité de l'Etat à protéger ses citoyens. Mais nous ajouterons aussi que dans le cas de risques globaux, ils interrogent également la souveraineté des Etats ainsi que leur réputation sur la scène internationale à travers leur capacité à contribuer à la santé, comme bien public mondial.

Mais cet exemple montre également que, loin d'être figés dans une supposée spécificité culturelle, les modes de gouvernance «locaux» sont extrêmement dynamiques et capables d'évolutions rapides. Les autorités vietnamiennes se sont adaptées à la gestion des incertitudes dans un système plus ouvert et globalisé. Certes cet apprentissage s'est fait en partie sous la pression et les contraintes exercées par la communauté internationale. De nombreux acteurs, et avec eux un ensemble de normes et de valeurs, ont renforcé leur influence dans ce pays à l'occasion de l'émergence du virus : le groupe agro-industriel thaillandais Charoen Pokphand a renforcé sa position sur le marché des volailles, les normes de l'OIE s'appliquent désormais à l'évaluation des services vétérinaires vietnamiens. Et, selon un expert international qui traque les nouveaux virus de grippe en Asie depuis des années, le virus $\mathrm{H} 5 \mathrm{~N} 1$ a agi « comme un hameçon » auquel le Vietnam a mordu, en s'impliquant désormais dans la coopération internationale contre les maladies émergentes. Pourtant:

"Le Vietnam joue le jeu parce qu'il en tire des bénéfices. C'est vrai qu'ily a des conditions à l'aide étrangère. Mais les bailleurs de fonds sont parfois naiifs... » (expert du Comité national de lutte contre la grippe aviaire, NSC).

Car derrière ces contraintes se profilent aussi des opportunités que le Vietnam a su exploiter, faisant preuve d'une grande habilité à entrer dans le jeu de la gouvernance internationale tout en gardant à distance cette communauté internationale, à détourner l'image d'un porteur d'une menace pandémique pour devenir un bon citoyen global, et au niveau local à consolider l'héritage communiste tout en prônant un plus grand contrôle par le marché de la santé publique.

Cette analyse souhaite montrer que la globalisation de la gouvernance sanitaire ne doit amener à négliger la complexité du local, la prise en compte des cultures nationales ne doit masquer les dynamiques locales, et les contraintes ne doivent masquer les opportunités indissociablement liées aux risques.

\section{Bibliographie}

Agrifood Consulting International (2007) Poultry sector rebabilitation project: The economic impact of highly pathogenic avian influenza - Related biosecurity policies on the Vietnamese poultry sector, Prepared for FAO and WHO, Bethesda, Maryland, 254 p.

Beck U. (2001) La société du risque. Sur la voie d'une autre modernité, Paris, Coll. Alto. Aubier, $521 \mathrm{p}$. 
Borraz O. (2008) Les politiques du risque, Paris, Presses de Sciences Po, 294 p.

Bush W.G. (2005) Discours au Sommet Mondial de l'ONU. 14 septembre 2005, Disponible sur http://www.un.org/webcast/summit2005/statements/usa050914 eng2.pdf, (consulté le 4 février 2010)

Callon M. (1986) Éléments pour une sociologie de la traduction. La domestication des coquilles Saint-Jacques dans la Baie de Saint-Brieuc, L'Année Sociologique 36, 169-208.

Congressional Research Service (2006) (updated) U.S. and international responses for the global spread of avian flu: Issues for congress, The Library of Congress, RL33219, $46 \mathrm{p}$.

Delquigny T., Edan M., Nguyen Dinh Hoan, Pham Trung Kien and Gautier P. (2004) Evolution and impact of avian influenza epidemic and description of the avian production in Vietnam. Final report, Hanoi, Association Vétérinaires Sans Frontières, 119 p.

Demortain D. (2009) Rendre transférable plutôt que diffuser. Les experts scientifiques et l'histoire de la norme HACCP, in: Normer le monde, Schemeil Y. et Eberwein W.D. (éds), Paris, L'Harmattan, Logiques Politiques, 131-152.

Do Hien (2007) Les relations internationales du Viêt Nam depuis 1991. La décennie des grands changements au service d'une stratégie pour la sécurité et le développement, in: Viet Nam contemporain, Dovert S., Tréglodé B. (dir.), Paris, Irasec, 171-186.

Douglas M., Wildavsky A. (1983) Risk and culture: An essay on the selection of technological and environmental dangers, Berkeley, University of California Press, $221 \mathrm{p}$.

Gilbert C. (2002) La fin des risques? Quaderni 48(1), 111-120.

Gilbert C. (dir) (2007) Les crises sanitaires de grande ampleur: un nouveau défi? Paris, La Documentation Française, INHES, 64 p.

Godard O., Henry C., Lagadec P. et Michel-Kerjan E. (2002) Traité des nouveaux risques, Paris, Gallimard, Coll. Folio-actuel, 620 p.

Guénel A., Klingberg S. (2010) Press coverage of bird flu epidemic in Vietnam, in: Liberalizing, feminizing and popularizing bealth communications in Asia, Liew Kai Khiun (eds), Farnham, Ashgate, 77-94.

Haas P.M. (1992) Epistemic communities and international policy coordination, International Organization 46(1), 1-35.

Hassenteufel P. (2005) De la comparaison internationale à la comparaison transnationale, Revue Française de Science Politique 55(1), 113-132.

Hinrichs J., Otte J., Rushton J. (2010) Technical, epidemiological and financial implications of large-scale national vaccination campaigns to control HPAI H5N1, CAB Reviews Perspectives in Agriculture, Veterinary Science, Nutrition and Natural Resources 5(21), 1-20.

Löfstedt R.E., Vogel D. (2001) The changing character of regulation: A comparison of Europe and the United States, Risk Analysis 21(3), 399-416. 
Millstone E. (2009) Science, risk and governance: Radical rhetoric and the realities of reform in food safety governance, Research Policy 38, 624-636.

MARD - Ministry of Agriculture and Rural Development -, MOH - Ministry of Health - (2010) Avian and pandemic influenza. Vietnam's experience, Hanoi, MARD, $54 \mathrm{p}$.

MARD - Ministry of Agriculture and Rural Development - (2007) Provincial summary reports on implementing Prime Minister decision $n^{\circ}$ 394/QD-TTG, Hanoi, MARD, $108 \mathrm{p}$.

MARD - Ministry of Agriculture and Rural Development - (2006) Project centralisation and industrialisation of poultry farming, slaughtering and processing for the period 2006-2015, Hanoi, MARD.

OCDE - Organisation de Coopération et de Développement Economiques - (2003) Les risques émergents au $X X^{e}$ siècle. Vers un programme d'action, Paris, OCDE, $332 \mathrm{p}$.

OMS - Organisation Mondiale de la Santé - (2005) Communication en cas de flambée épidémique, Disponible sur http://www.who.int/csr/don/Avian_French.pdf (consulté le 4 février 2010).

OMS - Organisation Mondiale de la Santé - (2007) Rapport sur la santé dans le monde 2007. Un avenir plus sûr. La sécurité sanitaire mondiale au XXe siècle, OMS, 67 p.

OMS - Organisation Mondiale de la Santé - (2010) H5N1, Avian Influenza: Timelines of major events (update 4 January 2010), Disponible sur

http://www.who.int/csr/disease/avian_influenza/Timeline_10_01_04.pdf (consulté le $1^{\text {er }}$ avril 2010).

OIE - Organisation Mondiale de la Santé Animale - (2009) One world, One health, Bulletin de l'OIE 2, Disponible sur http://www.oie.int/eng/publicat/BULLETIN\%20PDF/Bull\%202009-2-ENG.pdf (consulté le 4 février 2010).

Pfeiffer D.U., Minh Phan Q., Martin V., Epprecht M. and Otte M.J. (2007) An analysis of the spatial and temporal patterns of highly pathogenic avian influenza occurrence in Vietnam using national surveillance data, The Veterinary Journal 174 (2), 302-309.

Renn O. (2008) Risk governance: Coping with uncertainty in a complex world, London Earthscan, Risk in Society Series, 455 p.

Scoones I., Forster P. (2008) The international response to bighly pathogenic avian influenza: Science, policy and politics, Brighton, STEPS Center, $57 \mathrm{p}$.

Taylor-Gooby P.F., Zinn J.O. (eds), (2006) Risk in social science, Oxford, Oxford University Press, $264 \mathrm{p}$.

Vu Tuong (2009) The Political Economy of Avian Influenza Response and Control in Vietnam, STEPS Working Paper 19, Brighton: STEPS Centre. 